\title{
Src promotes cutaneous wound healing by regulating MMP-2 through the ERK pathway
}

\author{
XUE WU ${ }^{*}$, LONGLONG YANG ${ }^{*}$, ZHAO ZHENG $^{*}$, ZHENZHEN LI, JIHONG SHI, YAN LI, \\ SHICHAO HAN, JIANXIN GAO, CHAOWU TANG, LINLIN SU and DAHAI HU \\ Department of Burns and Cutaneous Surgery, Xijing Hospital, \\ The Fourth Military Medical University, Xi'an, Shaanxi 710032, P.R. China
}

Received June 29, 2015; Accepted January 22, 2016

DOI: $10.3892 /$ ijmm.2016.2472

\begin{abstract}
Wound healing is a highly orchestrated, multistep process, and delayed wound healing is a significant symptomatic clinical problem. Keratinocyte migration and re-epithelialization play the most important roles in wound healing, as they determine the rate of wound healing. In our previous study, we found that $\mathrm{Src}$, one of the oldest proto-oncogenes encoding a membrane-associated, non-receptor protein tyrosine kinase, promotes keratinocyte migration. We therefore hypothesized that Src promotes wound healing through enhanced keratinocyte migration. In order to test this hypothesis, vectors for overexpressing Src and small interfering RNAs (siRNAs) for silencing of Src were used in the present study. We found that the overexpression of Src accelerated keratinocyte migration in vitro and promoted wound healing in vivo without exerting a marked effect on cell proliferation. The extracellular signalregulated kinase (ERK) and c-Jun N-terminal kinase (JNK) signaling pathways play important roles in Src-accelerated keratinocyte migration. Further experiments demonstrated that Src induced the protein expression of matrix metalloproteinase-2 (MMP-2) and decreased the protein expression of E-cadherin. We suggest that ERK signaling is involved in the Src-mediated regulation of MMP-2 expression. The present study provided evidence that Src promotes keratinocyte migration and cutaneous wound healing, in which the regulation of MMP-2 through the ERK pathway plays an important role, and thus we also demonstrated a potential therapeutic role for Src in cutaneous wound healing.
\end{abstract}

Correspondence to: Professor Linlin Su or Professor Dahai Hu, Department of Burns and Cutaneous Surgery, Xijing Hospital, The Fourth Military Medical University, 127 Changle West Road, Xi'an, Shaanxi 710032, P.R. China

E-mail: xjburnlab@126.com

E-mail: zhengshangxuew@163.com

*Contributed equally

Key words: Src, wound healing, keratinocyte migration, extracellular signal-regulated kinase, matrix metalloproteinases

\section{Introduction}

Cutaneous wound healing is a dynamic and well-ordered biological process that involves three critical steps: inflammation, re-epithelialization and tissue remodeling (1). The re-epithelialization process plays the most significant role and requires the proper migration and proliferation of keratinocytes at the periphery of the wound (2). Keratinocyte migration is a complex process that involves the serial activation of kinases and cytokines. Src, one of the proto-oncogenes encoding a membrane-associated, non-receptor protein tyrosine kinase, has been implicated in the regulation of a wide range of cellular functions, including cell proliferation and cell migration (3). In normal and oncogenically transformed cells, it has ben proposed that Src family kinases regulate cell migration (4-6). Additionally, it has been demonstrated that Src is essential for wound healing in Drosophila as well as wound responses and tissue regeneration in zebrafish $(7,8)$. Another study has reported that the activation of Src promotes wound healing, whereas the inactivation of Src inhibits wound closure in mouse corneal epithelial cells (9). However, it remains unclear whether the role of Src in cutaneous wound healing is related to the regulation of keratinocyte migration.

Keratinocyte migration also depends on the loss of cell-matrix and cell-cell adhesion; the ability of keratinocytes to detach from the underlying basal lamina and migrate through the fibrin and extracellular matrix (ECM) meshwork of the wound is important to the re-epithelialization process. Matrix metalloproteinases (MMPs), which degrade different components of the ECM, are essential for keratinocyte migration. Following cutaneous injury, MMP expression is temporally and spatially regulated in the wound; this helps to initiate and maintain keratinocyte migration and is necessary for wound re-epithelialization (10-12). Human keratinocytes synthesize and secrete mainly MMP-1, MMP-2, MMP-9 and MMP-10 (2). Several studies have linked the gelatinases, MMP-2 and MMP-9, contribute to cancer, infectious diseases, inflammation, vascular diseases and wound healing $(10,12,13)$. The mitogen-activated protein kinase (MAPK) signaling pathway has been implicated in MMP-2 expression in oral cancer cells (14). Moreover, E-cadherin is a protein which mediates cell-cell adhesion by forming homodimers on adjacent cells. Accordingly, these data prompted us to investigate 
the function of MAPK, MMPs and E-cadherin in the regulation of Src-mediated keratinocyte migration in wound healing.

We hypothesized that Src accelerates keratinocyte migration, at least in part, through MAPK, MMPs and E-cadherin. To test this hypothesis, keratinocytes and in vivo wounds were pre-treated with vector pcDNA3.1(+)-Src for overexpressing Src and Src-specific small interfering RNA (siRNA) for the silencing of Src, and then the effects on MAPK activation, cell migration, E-cadherin, MMPs and wound healing were determined. Our in vitro study found that Src promoted keratinocyte migration through the upregulation of MMP-2 and the downregulation of E-cadherin, and that the extracellular signal-regulated kinase (ERK) pathway was involved in the Src-induced increase of MMP-2. Our in vivo experiments showed that Src accelerated wound healing. Thus, the present study offers valuable insights into the molecular mechanisms responsible for keratinocyte migration and wound healing, and it provides a rationale for the therapeutic effect of Src on cutaneous wound healing.

\section{Materials and methods}

Animals and antibodies. Adult male Sprague-Dawley (SD) rats $(\mathrm{n}=50)$ weighing $220-250 \mathrm{~g}$ were purchased from the Center of Experimental Animals at the Fourth Military Medical University (FMMU; Xi'an, China). The experiments were conducted in accordance with the Guide for the Care and Use of Laboratory Animals of the FMMU, and all experimental protocols used in this study were approved by the Animal Care Committee of the FMMU.

Cell culture. All human tissues were obtained from 4 patients (mean age, 30 years) at Xijing Hospital (Xi'an, China). Hypertrophic scar and surrounding normal skin tissues were obtained from the same patients. Prior to the experiment, all patients were informed about the purpose of the study as well as the procedures, and voluntarily agreed to provide tissue. Written consent was obtained from all participants, and all protocols were approved by the Ethics Committee of Xijing Hospital, which is affiliated with the FMMU. Briefly, the epidermal layer of human keratinocytes was separated from the dermis and placed into a sterile $15-\mathrm{ml}$ conical tube containing $2 \mathrm{ml} 0.05 \%$ trypsin-EDTA. The cells were incubated at $37^{\circ} \mathrm{C}$ for approximately $15 \mathrm{~min}$, during which time the cells were triturated using a 2-ml pipette every 2-3 min to aid cell dissociation. The cells were centrifuged at $180 \mathrm{xg}$ for $7 \mathrm{~min}$ at room temperature. After resuspension, the primary keratinocytes were seeded in $75 \mathrm{~cm}^{2}$ tissue culture flasks at a density of approximately $3 \times 10^{6}$ cells/ flask in $15 \mathrm{ml}$ keratinocyte serum-free medium (KSFM) with the addition of bovine pituitary extract (both from Gibco, Grand Island, NY, USA), and $1 \%$ antibiotics (penicillin-streptomycin, both $100 \mathrm{U} / \mathrm{ml}$ ). The medium was changed every 2 days until the cells reached confluence, and the keratinocytes were cultured continuously until use.

Westernblotanalysis. Thekeratinocyteswerewashed withice-cold phosphate-buffered saline (PBS) and lysed using RIPA buffer supplemented with protease and phosphatase inhibitor mixtures (Heart Biological Technology Co.,Ltd., Xi'an, China) on ice. The lysates were separated by centrifugation at $4^{\circ} \mathrm{C}$ and $14,000 \mathrm{x}$ for $10 \mathrm{~min}$. Subsequently the protein concentration was determined using a bicinchoninic acid (BCA) assay (Pierce, Rockford, IL, USA). Fifty micrograms of total protein was subjected to sodium dodecyl sulfate-polyacrylamide gel electrophoresis (SDS-PAGE) and transferred to PVDF membranes (Millipore Corp., Bedford, MA, USA). After blocking with 5\% non-fat milk, the membranes were incubated with mouse anti-Src (1:200, sc-8056; Santa Cruz Biotechnology, Inc., Santa Cruz, CA, USA), rabbit anti-ERK1/2 (4695), anti-phosphorylated (p-)ERK1/2 (4370), anti-c-Jun N-terminal kinase (JNK) (9258), anti-p-JNK (4668), anti-p38 (8690), anti-p-p38 (4511) (all at 1:1,000 dilution; Cell Signaling Technology, Inc., Danvers, MA, USA), mouse anti-MMP-2 (ZS-135950), goat anti-MMP-9 (ZS-68400, both at 1:200 dilution; Zhongshan Jinqiao Biological Technology, Co. Ltd., Beijing, China), mouse anti-E-cadherin (1:1000, 610181; BD Biosciences, Franklin Lakes, NJ, USA), goat anti-actin (1:200, sc-1616; Santa Cruz Biotechnology, Inc.) overnight at $4^{\circ} \mathrm{C}$. The following day, the membranes were incubated with horseradish peroxidaseconjugated secondary antibodies $(1: 3,000)$ at $37^{\circ} \mathrm{C}$ for $1 \mathrm{~h}$. The immunoreactive proteins were then visualied using ECL western blotting detection reagent (Millipore Corp., Billerica, MA, USA) and detected using the MultiImage Light Cabinet filter positions (Alpha Innotech Corp., San Leandro, CA, USA).

Plasmid vector for Src overexpression and siRNA for silencing of Src expression. Full-length human Src cDNA was amplified by reverse transcription-polymerase chain reaction (RT-PCR) from normal skin RNA. The PCR products were digested with Nhe I and $K p n I$, and subcloned into the eukaryotic expression vector, pcDNA3.1(+) (GeneCopoeia, Rockville, MD, USA). The recombinant pcDNA3.1(+)-Src plasmid (GeneCopoeia) was confirmed by sequencing. The keratinocytes were plated in $60 \mathrm{~cm}^{2}$ wells for $24 \mathrm{~h}$ prior to transfection. Thereafter, the cells were transfected with either $5 \mu \mathrm{g}$ pcDNA3.1(+)Src plasmid or the empty vector (GeneCopoeia) using Lipofectamine 2000 transfection reagent (Invitrogen, Carlsbad, CA, USA). The siRNA (80 nM; Thermo Fisher Scientific, Waltham, MA, USA) duplex sequence was as follows: sense, 5'CGAGUGCCUUAUCCAAGAATT-3'. The cells transfected with scrambled siRNA (Thermo Fisher Scientific) (sense, 5'UUCUUGGAUAAGGCACUCGTT-3'), which were the mock group, were also transfected using Lipofectamine 2000 transfection reagent. Western blot analysis was performed after an additional $48 \mathrm{~h}$.

Flow cytometric analysis. Cell cycle distribution was analyzed by flow cytometry (FACSAria; BD Biosciences). Primary keratinocytes were divided into the following groups: control, pcDNA3.1(+), pcDNA3.1(+)-Src, mock and also the Src-siRNA transfected groups. Twenty-four hours after transfection, the cells were harvested, rinsed with PBS, fixed with $95 \%$ (v/v) ice-cold ethanol and resuspended in staining buffer containing FITC-Annexin $\mathrm{V}$ and propidium iodide (PI). The mixture was then incubated in the dark at room temperature for $15 \mathrm{~min}$. The DNA contents of the stained nuclei were analyzed, and the number of cells in each cycle phase was calculated.

In vitro cell migration assays. In the present study, the keratinocytes transfected with pcDNA3.1(+)-Src plasmid ,Src-siRNA, pcDNA3.1(+), scrambled siRNA (mock group) and untransfected cells (as control), were grown to confluence 

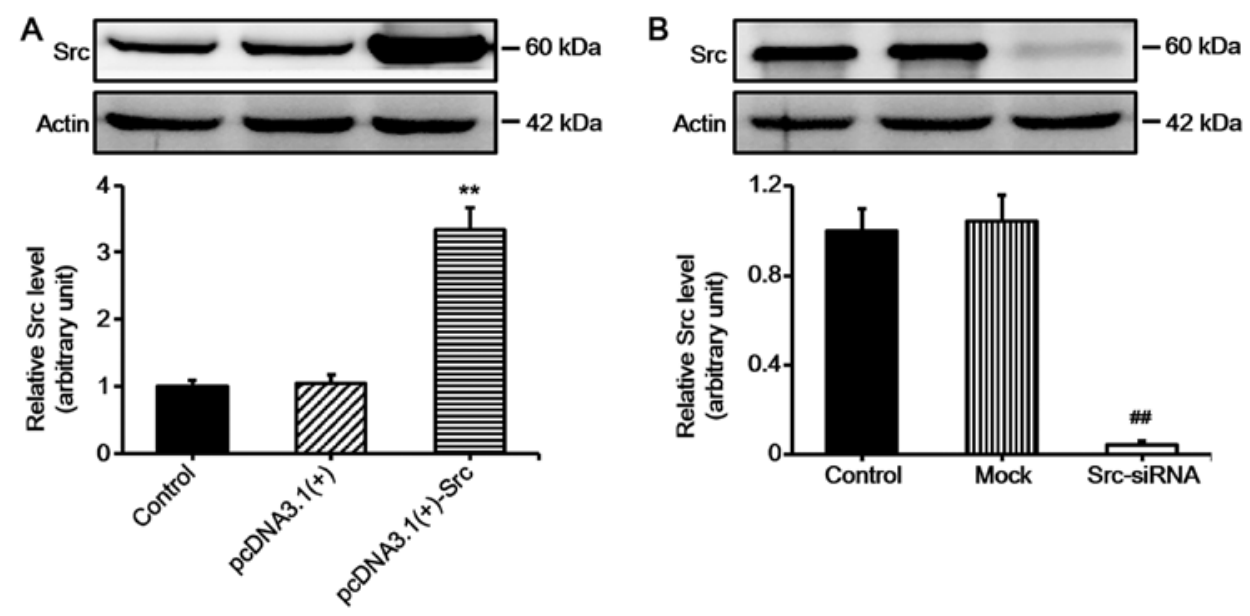

Figure 1. Western blot analysis of Src expression in keratinocytes following transfection. (A) Keratinocytes were transfected with pcDNA3.1(+)-Src for overexpressing Src and (B) Src-siRNA was used for silencing Src. Controls used were untransfected cells (control), pcDNA3.1(+) and mock. Mock, cells transfected with scrambled siRNA. Bars represent the means $\pm \mathrm{SEM} ; \mathrm{n}=4$ tissue samples. ${ }^{* *} \mathrm{P}<0.01$ vs. pcDNA3.1(+) group, ${ }^{\# \#} \mathrm{P}<0.01$ vs. mock group.

in 12 -well plates in $\mathrm{KSFM}$ containing $10 \mu \mathrm{g} / \mathrm{ml}$ mitomycin $\mathrm{C}$ (Invitrogen) for $1 \mathrm{~h}$ to completely inhibit cell proliferation. A straight scratch was made on the keratinocytes using a P200 pipette tip. The cells were then washed with PBS three times, and further cultured in KSFM. After incubating for 0,12 and $24 \mathrm{~h}$, the gap width of scratch re-population was measured and recorded, and then compared with the initial gap size at $0 \mathrm{~h}$. Using the ImageJ image processing program, the size of the denuded area was determined at each time point from the digital images. Where indicated, the cells were treated with JNK inhibitor (SP-600125 at $30 \mu \mathrm{M}$; Sigma-Aldrich, St. Louis, MO, USA) or the ERK inhibitor (PD-98059 at $30 \mu \mathrm{M}$; Beyotime, Haimen, China) $1 \mathrm{~h}$ prior to experiments.

Immunocytofluorescence analysis. For immunocytofluorescence analysis, keratinocytes were fixed with $4 \%$ paraformaldehyde. Cells were permeabilized with Triton X-100 and then blocked with BSA. Cells were incubated with anti-E-cadherin $(1: 100,610181$; BD Biosciences $)$ at $4^{\circ} \mathrm{C}$ overnight and with CY3-555 IgG antibody (A28180; Invitrogen) at $37^{\circ} \mathrm{C}$ for $1 \mathrm{~h}$ on the following day. DAPI was used for nuclear staining. Images were captured by an FSX100 microscope (Olympus, Tokyo, Japan)

In vivo wound closure assay. Following anesthetization, a $1.0 \times 1.0-\mathrm{cm}^{2}$ full-thickness wound was punched onto the back of male SD rats. To evaluate the effect of Src on wound healing, $20 \mu \mathrm{g}$ pcDNA3.1(+)-Src plasmids and $400 \mathrm{nM}$ Src-siRNA duplexes were topically transfected into the epidermis surrounding the wounds at four sites on days 1, 5, 9, 13 and 17 for a total of five doses. The same quantity of empty plasmid and scrambled siRNA were used as controls. The process of wound healing was digitally photographed and recorded. Wound area measurement was performed by digital planimetry using ImageJ software. The relative residual wound area was calculated as the ratio between the residual wound area at a given time point and the original wound area $\mathrm{x} 100 \%$. At least 10 rats were used for each time point examined. The average unhealed wound gap was defined as the distance between the advancing edges of epidermal keratinocyte migration. All images were captured with the FSX100 microscope; multiple overlapping pictures were used to artificially reconstitute the entire wounds.

Statistical analysis. Each experiment was repeated at least three times, and the data are presented as the means \pm SEM. Statistical differences between groups were analyzed by the Student's t-test or the Mann-Whitney U test as appropriate using SPSS 13.0. A P-value $<0.05$ was considered to indicate a statistically significant difference.

\section{Results}

Src promotes keratinocyte migration and does not markedly affect keratinocyte proliferation in vitro. Keratinocyte migration and proliferation determine the rate of wound re-epithelialization (1). In order to examine the role of Src in keratinocyte migration in vitro, we first constructed vector for overexpressing Src [pcDNA3.1(+)-Src] and siRNA for silencing Src (Src-siRNA). Western blot analysis confirmed the effective overexpression of Src and silencing of Src (Fig. 1). Subsequently, scratch wound healing assays were performed in order to evaluate the effects of overexpressing Src and silencing of Src on keratinocyte migration. The cells were pre-treated with mitomycin $\mathrm{C}$ for $1 \mathrm{~h}$ to inhibit proliferation prior to the scratch assay. The results demonstrated that keratinocytes overexpressing Src migrated into over $90 \%$ of the scratched area, pcDNA3.1(+)-Src group, while the Src-silenced keratinocytes migrated into only $30 \%$ of the area (Src-siRNA-transfected group), compared with the untransfected cells (as control) or negative vectors [mock and pcDNA3.1(+) group] at 24 h postscratching (Fig. 2A and B). Flow cytometric analysis showed that Src did not significantly affect the percentage of cells in the G1, G2, or S phase, indicating that Src did not exert a marked effect on cell proliferation (Fig. 2C). Taken together, these results suggest that Src promotes keratinocyte migration while exerting no marked effect on cell proliferation.

Src promotes cutaneous wound healing in vivo. Our in vitro experiment indicated that Src promotes keratinocyte migration. As keratinocyte migration plays a very important role in wound 

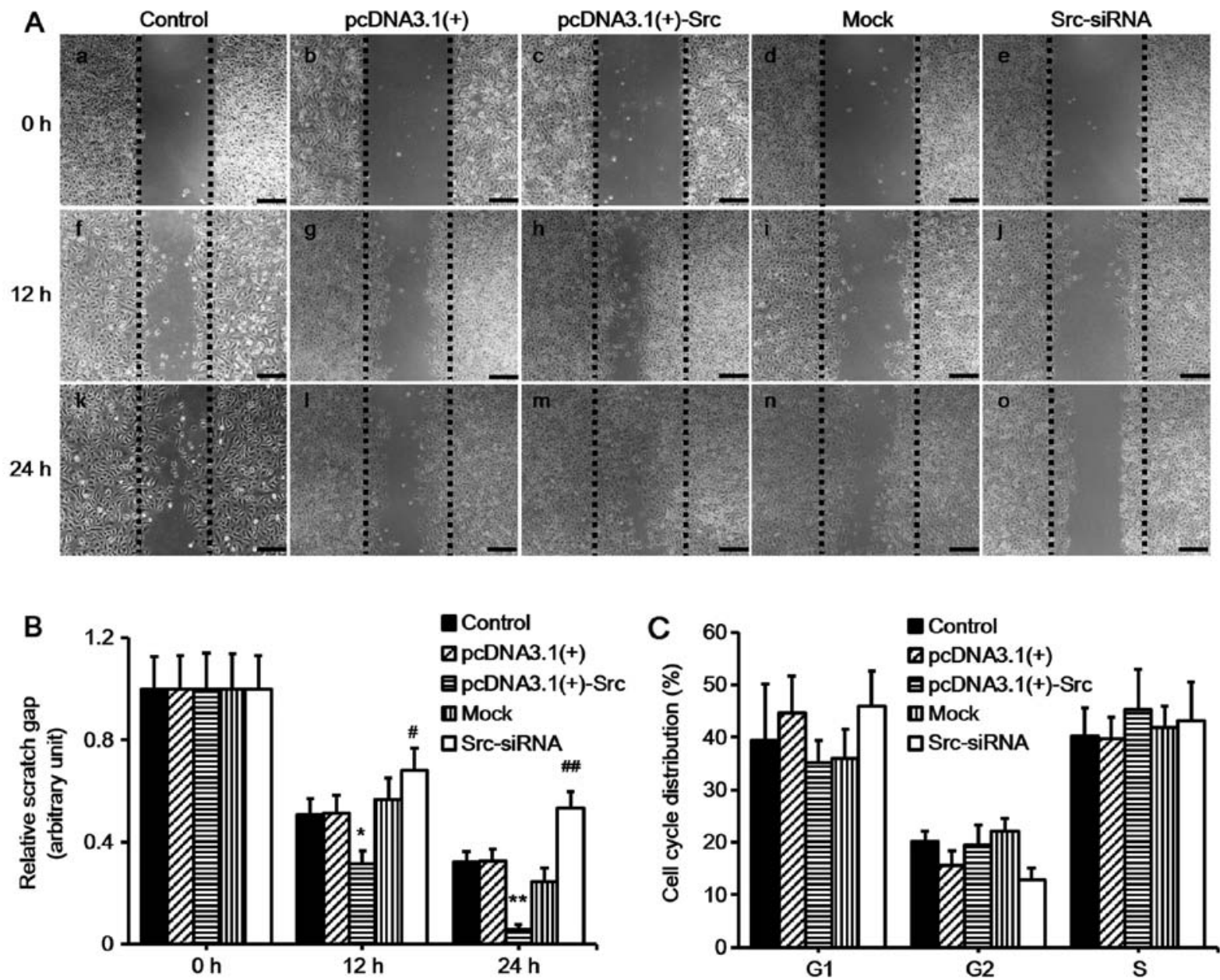

Figure 2. Effect of Src on keratinocyte migration in vitro. (A) Keratinocytes were transfected to overexpress Src [pcDNA3.1(+)-Src] or silence Src expression (Src-siRNA). Following $1 \mathrm{~h}$ of mitomycin $\mathrm{C}$ treatment, the cells were scratch-wounded with a micropipette tip ( $200 \mu \mathrm{l})$. Black dotted lines indicate the wound borders at the beginning of the assay and were recorded at 0,12 and $24 \mathrm{~h}$ post-scratching. Scale bar, $100 \mu \mathrm{M}$. (B) Relative scratch gap was calculated as the ratio of the remaining scratch gap at the given time point and the original gap at $0 \mathrm{~h}$. (C) Flow cytometric analysis showing the effect of Src on cell cycle distribution. Numbers of cells at G1, G2 and S phases were counted and the percentage was calculated. Controls used were untransfected cells (control), pcDNA3.1(+) and mock; mock, cells transfected with scrambled siRNA sequence. Bars represent the means $\pm \mathrm{SEM} ; \mathrm{n}=4$ tissue samples. "P<0.05 vs. pcDNA3.1(+) group, ${ }^{\#} \mathrm{P}<0.05$ vs. mock group, ${ }^{* *} \mathrm{P}<0.01$ vs. pcDNA3.1(+) group, ${ }^{\# \#} \mathrm{P}<0.01$ vs. mock group.

healing, we hypothesized that Src promotes wound healing in vivo. As in the previous experiment, the Src-overexpression vector, pcDNA3.1(+)-Src, and Src-siRNA were also applied to wounds of SD rats (data not shown). To test our hypothesis, we first established a rat model of full-thickness wound healing. The pcDNA3.1(+), pcDNA3.1(+)-Src, mock (scrambled siRNA) and Src-siRNA were injected at the wound edges on days 1, 5, 9, 13 and 17 post-wounding. Wound healing was analyzed daily and selected wound images are shown (Fig. 3A). The results showed that pcDNA3.1(+)-Src transfection significantly accelerated wound closure on 14 and 21 days, while it was observed that the wounds of the Src-siRNA-transfected rats healed at a slower rate on days 14 and 21, indicating that the initial wound healing in the Src group happened as early as day 7 post-wounding (Fig. 3). Taken together, these results suggest that overexpression of Src at the wound edge accelerates the process of wound healing.

ERK and JNK signaling are involved in Src-promoted keratinocyte migration. In the present study, in order to explore the role of the MAPK pathways in Src-regulated keratinocyte migration, we investigated the activation of MAPK pathways in keratinocytes overexpressing Src and Src-silenced keratinocytes. The results showed that the phosphorylation levels of JNK and ERK were significantly increased in keratinocytes overexpressing Src and decreased in Src-silenced keratinocytes, while the phosphorylation levels of $\mathrm{p} 38$ were relatively unchanged (Fig. 4). These results suggest that the JNK and ERK pathways participate in Src-promoted keratinocyte migration. To further confirm the important role of the JNK and ERK pathways, keratinocytes overexpressing Src were treated with ERK inhibitor (PD-98059 at $30 \mu \mathrm{M}$ ) or JNK inhibitor (SP-600125 at $30 \mu \mathrm{M}$ ) or both. The cells were pretreated with mitomycin $\mathrm{C}$ to inhibit cell proliferation prior to the scratch wound-healing assays. The results showed that the ERK inhibitor, PD-98059, and the JNK inhibitor, SP-600125, slowed down the course of scratch gap closure when administered separately, while the combination of the two further slowed the progression (Fig. 5). Thus, taken together, the results of these experiments indicate that JNK and ERK signaling play a crucial role in the process of Src-promoted keratinocyte migration. 


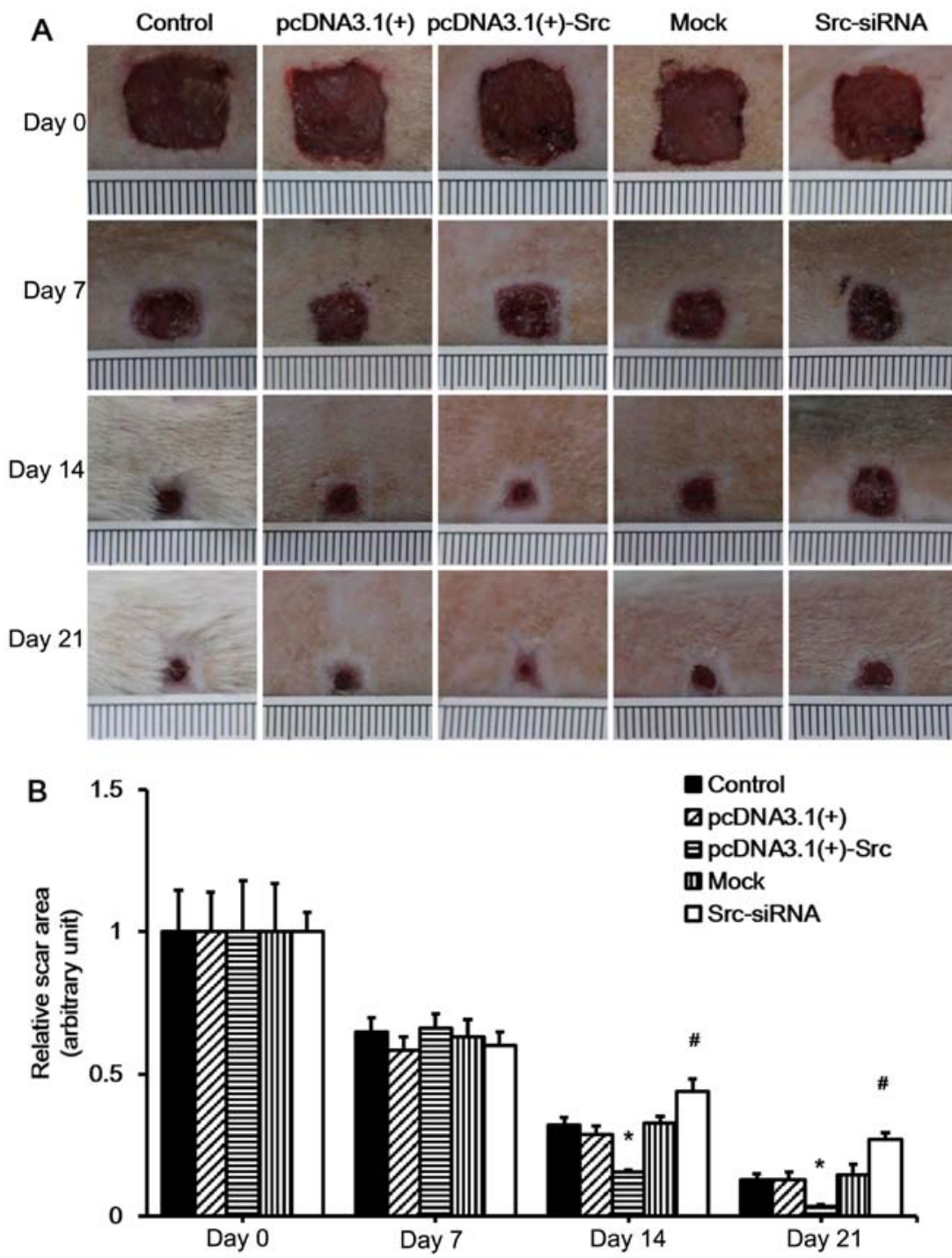

Figure 3. Effect of Src on rat skin wound healing. (A) Full-thickness excision wounds were made on the back of SD rats at day(d) 0 . The process of wound healing was digitally photographed on days $0,7,14$ and 21 post-wounding in the control, pcDNA3.1(+), pcDNA3.1(+)-Src, mock and Src-siRNA transfected groups. Ruler notches, $1 \mathrm{~mm}$. (B) The relative wound area was calculated as the ratio of the residual wound area at the given time-point and the original wound area on day 0. Control, saline; pcDNA3.1(+) and mock were used as negative controls; mock, cells transfected with scrambled siRNA. Bars represent the means $\pm \mathrm{SEM} ; \mathrm{n}=10$ rats/group. ${ }^{*} \mathrm{P}<0.05$ vs. pcDNA3.1(+) group, ${ }^{\#} \mathrm{P}<0.05$ vs. mock group.
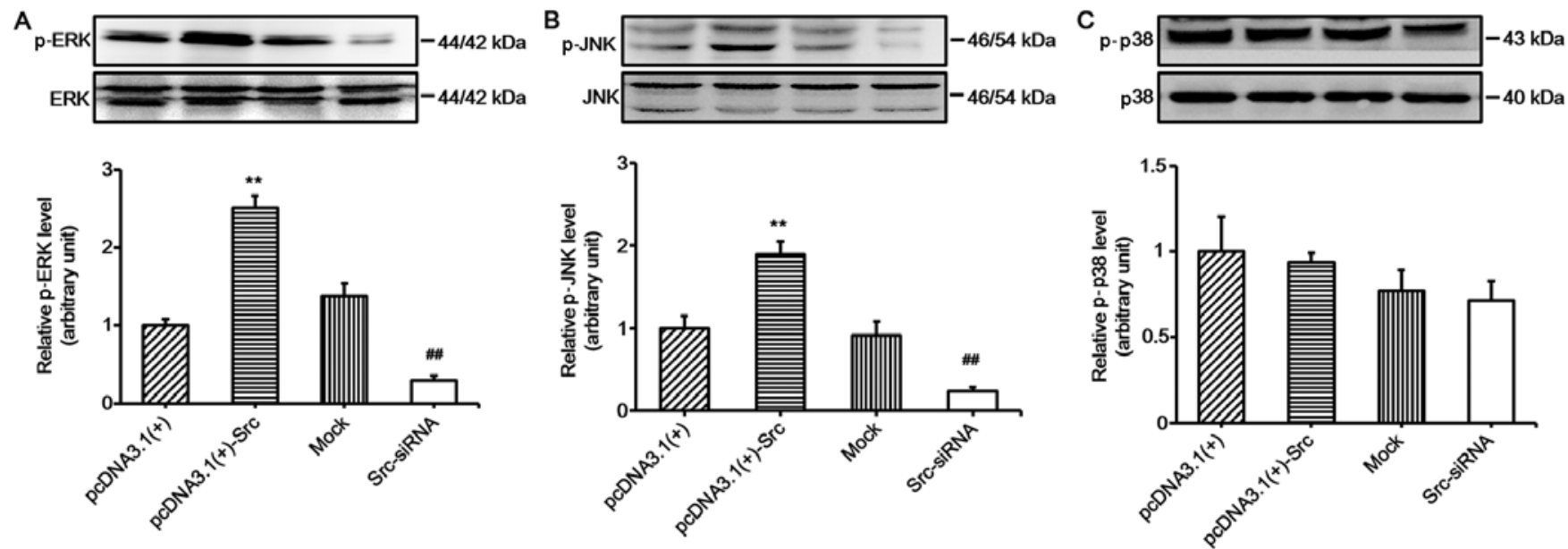

Figure 4. Western blot analysis of mitogen-activated protein kinase (MAPK) in keratinocytes after Src silencing or overexpression. Protein expression of (A) ERK, (B) JNK and (C) p38 after overexpressing Src [pcDNA3.1(+)-Src] or silencing Src expression (Src-siRNA). pcDNA3.1(+) and mock were used as negative controls; mock, cells transfected with scrambled siRNA. Bars represent the means $\pm \mathrm{SEM} ; \mathrm{n}=4 .{ }^{* *} \mathrm{P}<0.01$ vs. pcDNA3.1(+) group, ${ }^{\# \# / P}<0.01$ vs. mock group. 

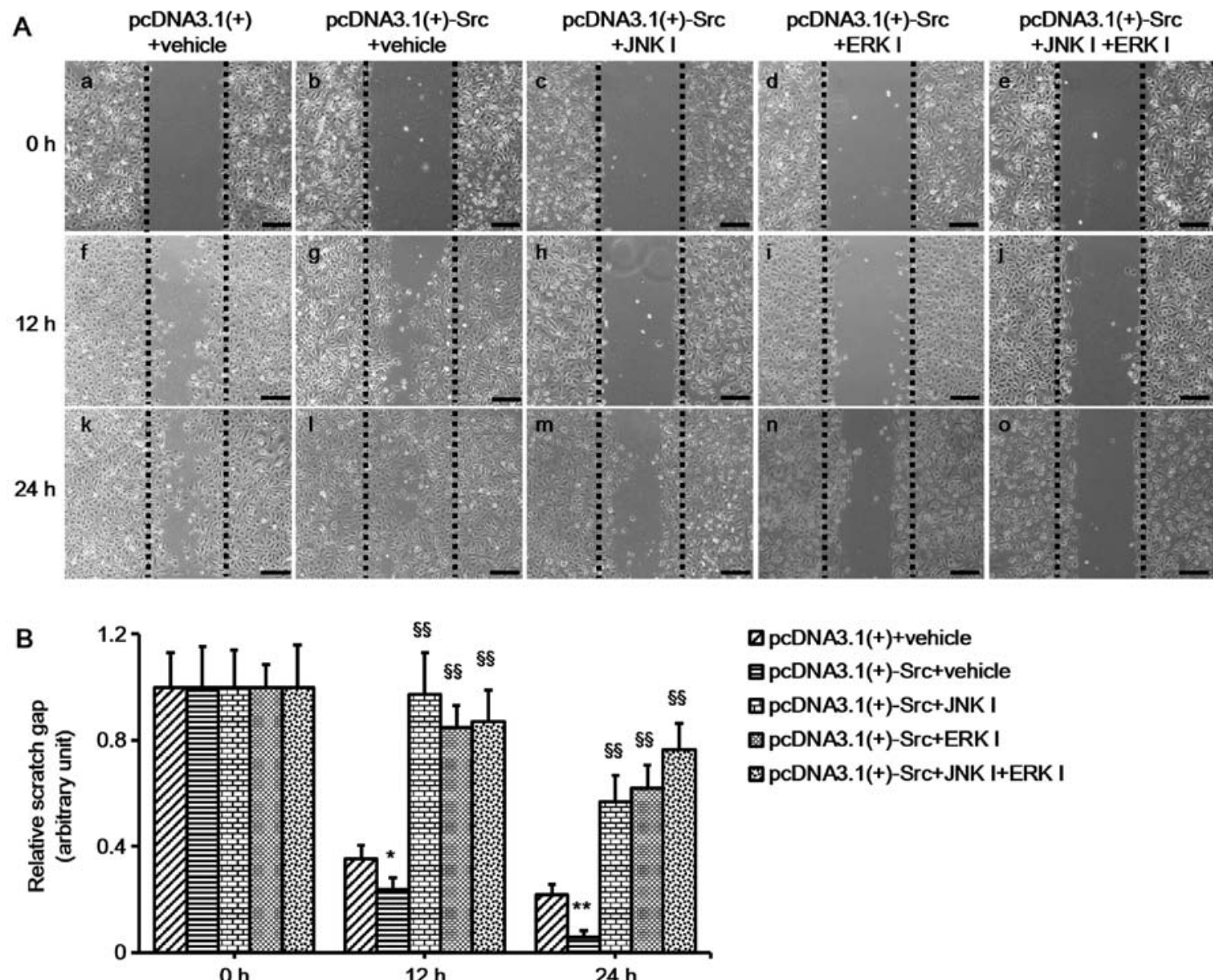

Figure 5. In vitro scratch assay following inhibition of extracellular signal-regulated kinase (ERK) and c-Jun N-terminal kinase (JNK) phosphorylation in Srcregulated keratinocytes. (A) Keratinocytes overexpressing Src were treated with ERK inhibitor (ERK I; PD-98059 at $30 \mu$ M) or JNK inhibitor (JNK I; SP-600125 at $30 \mu \mathrm{M}$ ) or both. Scratch wound migration assays were then performed on confluent keratinocytes. Black dotted lines indicate the wound borders at the beginning of the assay. Scale bar, $100 \mu \mathrm{M}$. (B) Relative scratch gap was calculated as the ratio of the remaining scratch gap at given time-point and the original gap at 0 h. Vehicle, water. Bars represent the means \pm SEM; $n=4$ tissue samples. ${ }^{*} \mathrm{P}<0.05$ and ${ }^{* *} \mathrm{P}<0.01$ vs. pcDNA3.1(+) group. ${ }^{\S \S} \mathrm{P}<0.01$ vs. pcDNA3.1(+)-Src group.

MMP-2 and E-cadherin are involved in Src-promoted keratinocyte migration. Many factors play an important role in keratinocyte migration, particularly MMPs and E-cadherin $(15,16)$. In order to examine the mechanism of Src-promoted keratinocyte migration, the expression levels of MMP-2, MMP-9 and E-cadherin were determined. The results demonstrated that the expression of MMP-2 was significantly increased in keratinocytes overexpressing Src, and decreased in Src-silenced keratinocytes compared with the untransfected cells (acting as control) or negative vectors, which were the mock and pcDNA3.1(+) groups. The expression of MMP-9 was not changed significantly. By contrast to the results noted for MMP-2, the expression levels of E-cadherin were markedly decreased in keratinocytes overexpressing Src and increased in Src-silenced keratinocytes (Fig. 6). Thus, our findings suggest that MMP-2 and E-cadherin participate in Src-promoted keratinocytes migration.

ERK signaling is involved in Src-induced changes in MMP-2 expression. Our results indicated that the ERK and JNK pathways played important roles in Src-promoted keratinocyte migration, and the expression of MMP-2 and E-cadherin changed during this process. In order to further elucidate whether ERK and JNK signaling played an important role in Src-induced expression changes in MMP-2 and E-cadherin, the keratinocytes were transfected with pcDNA3.1(+)-Src vector and then treated with the ERK inhibitor, PD-98059, or the JNK inhibitor, SP-600125, or both. The results demonstrated that PD-98059 alone significantly prevented Src-induced changes in MMP-2 protein expression. The combination of the two inhibitors did not abolish MMP-2 expression. Moreover, no significant change was detected in the protein expression of MMP-9. The protein expression of E-cadherin was almost unchanged when the inhibitors were used on their own or in combination, indicating that a different mechanism was involved in the regulation of E-cadherin (Fig. 7). Collectively, these results indicate that ERK signaling plays an important role in Src-induced changes in MMP-2 protein expression.

\section{Discussion}

Wound healing is a complex process which requires re-epithelialization (17). Keratinocyte migration is an essential process in wound healing, and involves the serial activation of kinases and cytokines. Studies have demonstrated that Src family members participate in the regulation of the cell cycle, cell 
A

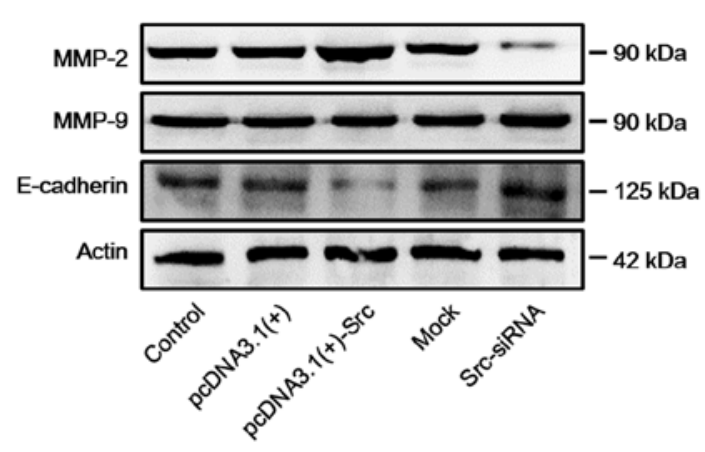

C
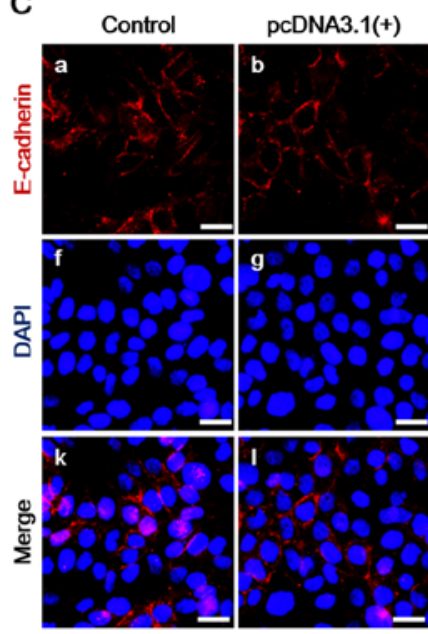
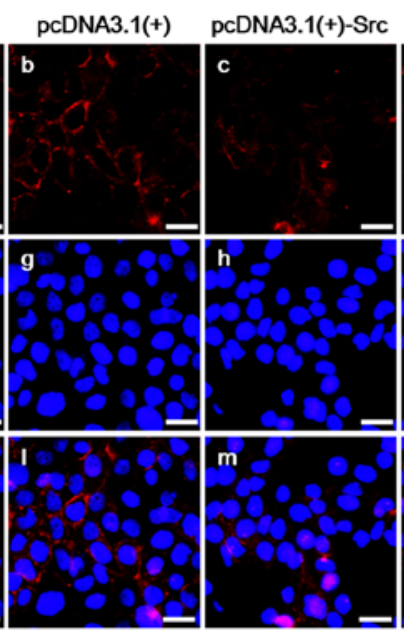

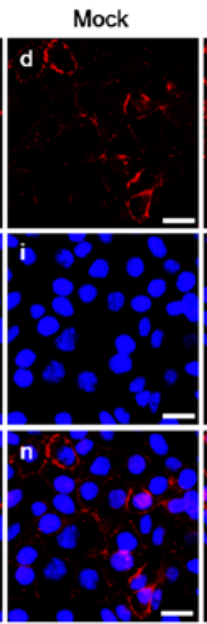

Src-siRNA
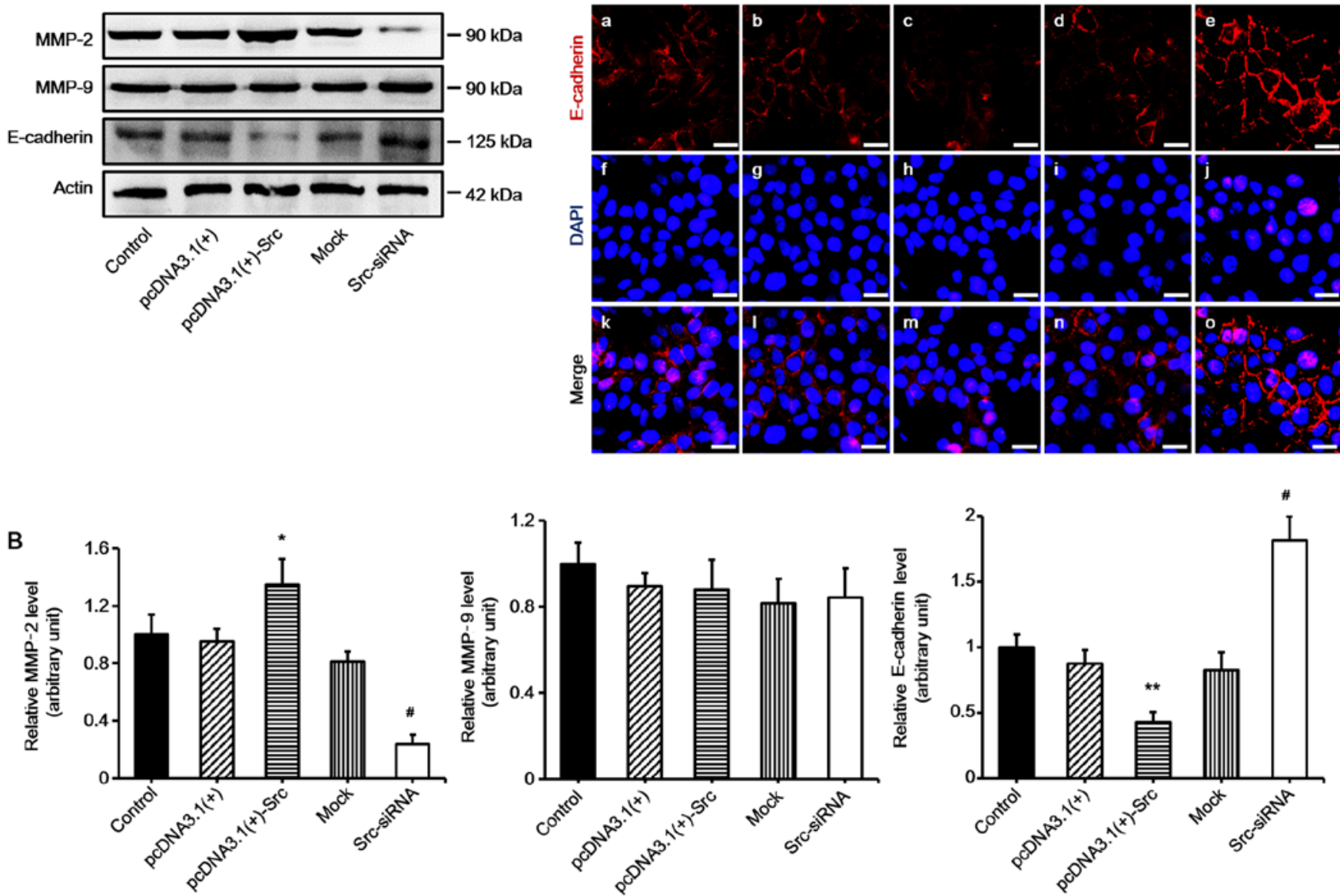

Figure 6. Involvement of matrix metalloproteinases (MMPs) and E-cadherin in Src-promoted keratinocyte migration. (A and B) Western blot analysis of MMP-2, MMP-9 and E-cadherin expression in keratinocytes following transfection with overexpressing Src [pcDNA3.1(+)-Src] or silencing Src (Src-siRNA), or the corresponding controls. Controls used were untransfected cells (control) and pcDNA3.1(+) and mock; mock, cells transfected with scrambled siRNA. Actin was used as a loading control. Bars represent the means \pm SEM of $n=4$ tissue samples. ${ }^{*} \mathrm{P}<0.05$, pcDNA3.1(+)-Src group vs. pcDNA $3.1(+)$ group, ${ }^{\# P}<0.05$, Src-siRNA vs. mock group ${ }^{* *} \mathrm{P}<0.01$, pcDNA3.1(+)-Src group vs. pcDNA 3.1(+) group. (C) Immunofluorescence staining of E-cadherin (red, Cy3-conjugated secondary antibody) in keratinocytes. Nuclei were visualized with DAPI (blue). Scale bar, $100 \mu \mathrm{M}$.

proliferation, migration, adhesion, and differentiation (18). In corneal epithelial cells, Src has been found to be activated along the leading edge of scratch-wounded cultures (9); however, the role of Src in keratinocytes has not previously been reported in depth. In the present study, we found that Src overexpression promoted keratinocyte migration and Src silencing inhibited keratinocyte migration without exerting a marked effect on keratinocyte proliferation in vitro (Figs. 1 and 2). The positive effects of Src on the migration of human keratinocytes suggest that Src plays a significant role in wound re-epithelialization. We also found that Src promoted wound healing in vivo in SD rats (Fig. 3). Taken together, these results demonstrate that the effect of Src on wound healing was mainly due to its role in keratinocyte migration.

MMPs are an important family of cell migration-related proteins which degrade different components of the ECM. Previous research has shown that MMPs also contribute to cell migration during wound healing (19). Several studies have demonstrated that following skin wounding, keratinocytes bind with type I collagen to stimulate an epidermal growth factor (EGF) receptor-dependent cascade resulting in the expression of MMPs (20-23). Previous research has revealed that exogenous treatment with MMP inhibitors delayed wound healing and re-epithelialization $(24,25)$. By contrast, the overexpression of MMPs was found to be detrimental to the wound healing process, and the expression level of MMPs was increased in chronic wounds $(26,27)$. These findings suggest that MMPs act positively and also negatively regulate wound healing processes, and collectively they indicate the importance of MMPs in wound healing. In addition, a previous study has shown that Src promotes cancer cell migration by increasing the levels of MMP-2 and MMP-9 (28). Consistent with this, in the present study we found that MMP-2 expression was increased during Src-induced accelerated keratinocyte migration, while the expression of MMP-9 was not changed significantly (Fig. 6). The inconsistent results may be related to the use of different cell lines in this previous study and our present study.

An essential condition for cell migration is the loosening of adherent junctions which facilitate cell-cell adhesion through homotypic binding between E-cadherin molecules on adjacent cells (29-31). Thus, E-cadherin is clearly another important protein in cell migration. A previous study has shown that Src kinase plays a crucial role in the maintenance of epithe- 
A
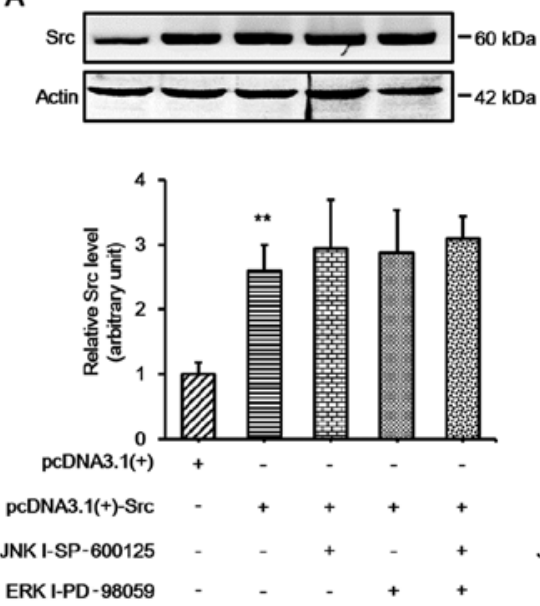

D
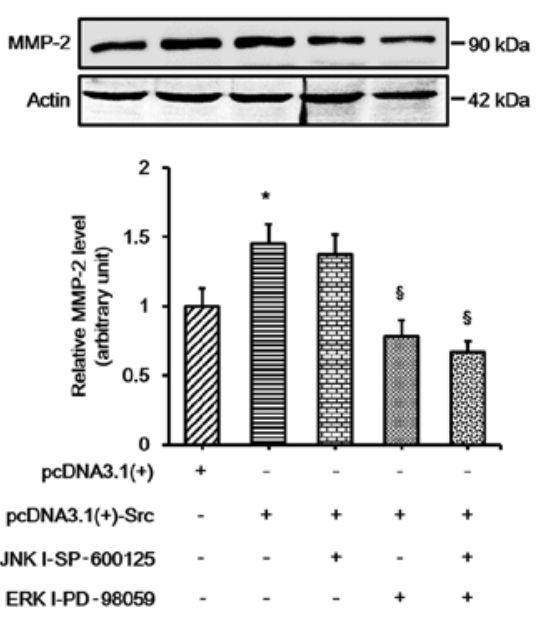

B
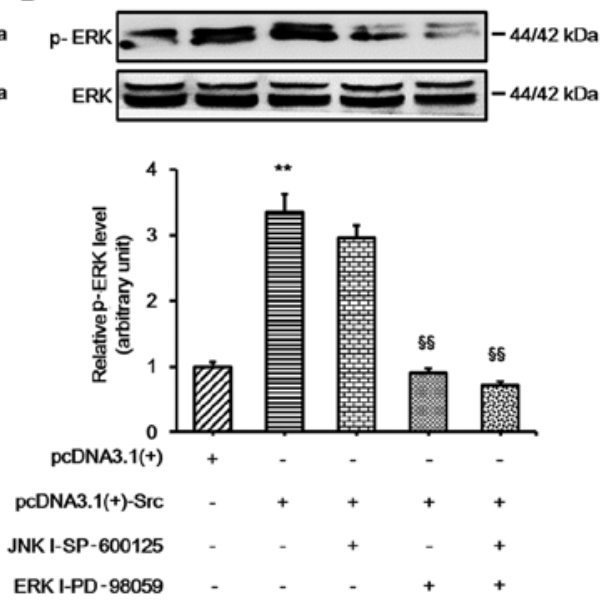

E
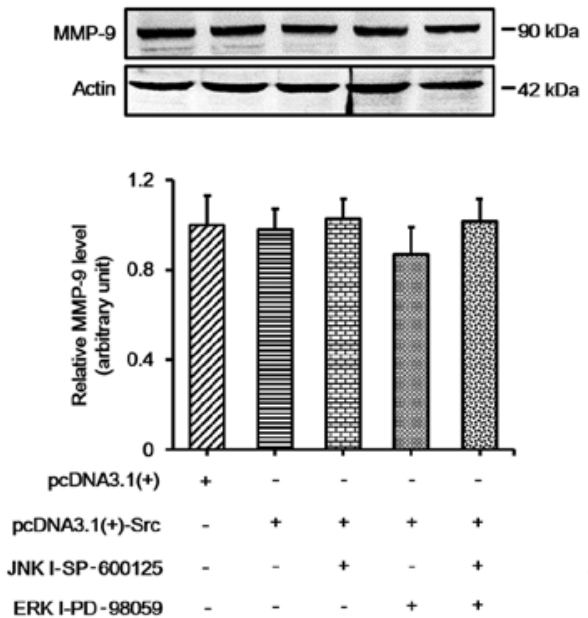

C
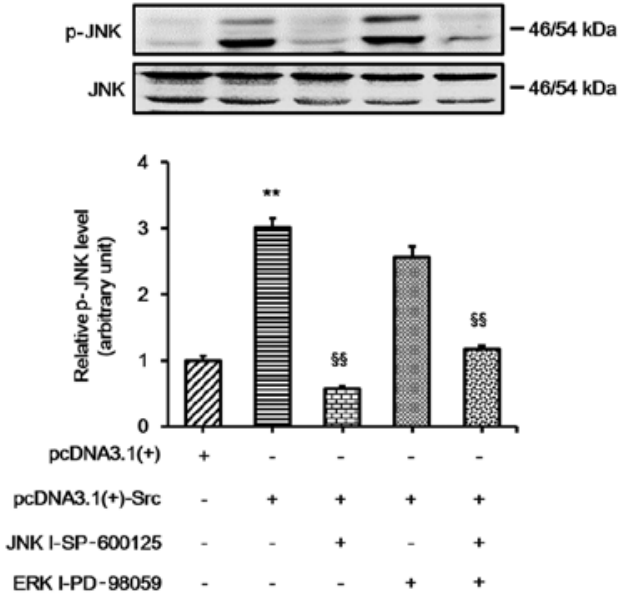

F
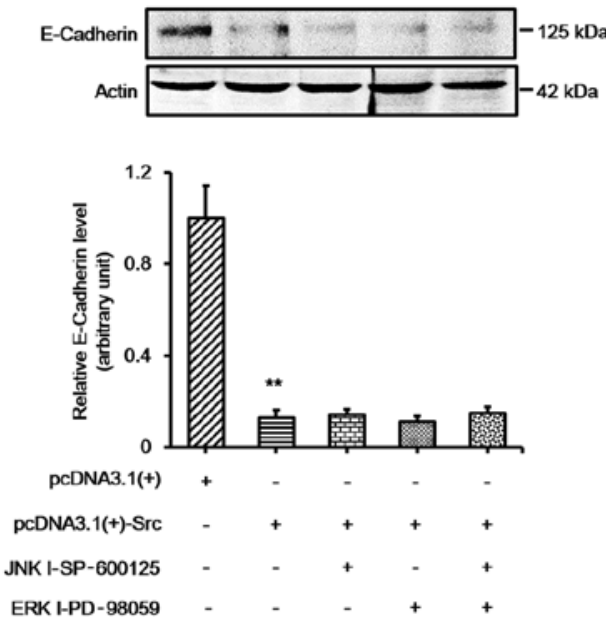

Figure 7. Involvement of extracellular signal-regulated kinase (ERK) signaling in Src-induced changes in MMP-2 expression. Western blot analysis showing the expression of (A) Src, (B) ERK, (C) c-Jun N-terminal kinase (JNK), (D) MMP-2, (E) MMP-9 and (F) E-cadherin in keratinocytes overexpressing Src treated with ERK inhibitor (ERK I; PD-98059 at $30 \mu \mathrm{M}$ ), JNK inhibitor (JNK I; SP-600125 at $30 \mu \mathrm{M}$ ) or both. Actin was used as a loading control. Bars represent the means \pm SEM of $n=4$ tissue samples. ${ }^{* *} \mathrm{P}<0.01$, pcDNA3.1(+)-Src group vs. pcDNA 3.1(+) group, ${ }^{*} \mathrm{P}<0.05$, pcDNA3.1(+)-Src group vs. pcDNA 3.1(+) group; ${ }^{\$ \$} \mathrm{P}<0.01$, JNK I - SP-600125 vs. pcDNA3.1(+)-Src group, ${ }^{\$ \$} \mathrm{P}<0.01$, ERK I-PD-98059 vs. pcDNA3.1(+)-Src group, ${ }^{\$ \$}$ P $<0.01$, JNK I - SP-600125 + ERK I-PD-98059 vs. pcDNA3.1(+)-Src group, ${ }^{\circledR} \mathrm{P}<0.05$, ERK I-PD-98059 vs. pcDNA3.1(+)-Src group, ${ }^{\circledR} \mathrm{P}<0.05$, JNK I - SP-600125 + ERK I-PD-98059 vs. pcDNA3.1(+)-Src group.

lial integrity by upregulating E-cadherin during epithelial morphogenesis (32). Similarly, it has previously been suggested that the regulation of E-cadherin expression in lung cancer is also linked to c-Src activity (33). Furthermore, several studies have demonstrated that the main role of Src in re-epithelialization is to control the adhesion of leading cells by regulating E-cadherin expression $(34,35)$. In the present study, we found that E-cadherin expression is partly regulated by Src (Fig. 6), and we suggest that a reduction of E-cadherin expression plays an important role in Src-promoted keratinocyte migration and accelerated wound healing. Our findings concur with previous research in cancer cell migration (36).

Src has been implicated in the regulation of signaling pathways associated with cell migration and proliferation, such as Akt, STAT3 phosphorylation and Ras activation (37-39). Previous research has indicated that Src is involved in the activation of MAPK in cancer cells and that it is considered a downstream target of Src signaling (40-42). MAPKs, including ERK1/2, JNK and p38, have all been implicated in the regulation of cell migration and proliferation (43-46). Downregulation of the ERK1/2/MAPK pathway leads to the decreased migration and proliferation of the cell (47-49). Notably, the inhibition of Src family kinases suppresses the activation of ERK1/2 (50). Additionally, it has been demonstrated that inhibition of the JNK/MAPK pathway decreases the migration of endothelial cells (51), and a correlation has been noted between the p38/MAPK pathway and cell migration into the wound along the wound edge (52). Thus, in the present study the phosphorylation levels of JNK, ERK1/2 and p38 were examined in keratinocytes overexpressing Src and Src-silenced keratinocytes. We found that the phosphorylation levels of JNK and ERK1/2 were significantly increased in keratinocytes overexpressing Src and decreased in Src-silenced keratinocytes, while the phosphorylation levels of p38 were relatively unchanged (Fig. 4). These findings suggest that the ERK1/2 and JNK pathways play an integral role in Src-regulated wound healing and also that the p38 pathway is not involved in this process. 
To further understand the mechanisms through which Src regulates wound healing through the ERK1/2 and JNK pathways, keratinocyte migration in vitro was evaluated after treating keratinocytes overexpressing Src with the ERK1/2 inhibitor PD-98059 or the JNK inhibitor SP-600125, or both. The protein expression levels of MMP-2, MMP-9 and E-cadherin were then assessed. We demonstrated that administration of the ERK1/2 inhibitor, the JNK inhibitor, or both, inhibited keratinocyte migration, and the inhibition of ERK1/2 signaling decreased MMP-2 expression; the inhibition of JNK activity did not markedly decrease MMP-2 expression (Figs. 5 and 7). No significant change was detected in the protein expression of MMP-9 and E-cadherin when inhibitors were used alone or in combination (Fig. 7). These findings suggest that the ERK1/2 pathway is involved in the process of Src-mediated upregulation of MMP-2. Activation of the MAPK pathway has been previously implicated in the regulation of MMP gene expression (14). Moreover, it has previously been reported that kaempferol reduces MMP-2 expression by downregulating ERK1/2 signaling pathways in oral cancer cells (14). The results of the present study reveal that ERK1/2 is involved in the Src-mediated regulation of MMP-2 expression, whereas the protein expression of E-cadherin is regulated through a different mechanism. In the future, in order to elucidate the mechanisms through which Src regulates wound healing via the ERK1/2 and JNK pathways, further investigation is clearly warranted.

In conclusion, in the present study we discovered that the overexpression of Src accelerates wound healing by promoting keratinocyte-migration, and that the mechanism appears to involve the upregulation of MMP-2 via the ERK1/2 pathway. These results provide us with a better understanding of the mechanisms responsible for wound healing and provide a novel therapeutic approach with which to improve wound healing.

\section{Acknowledgements}

The present study was supported by the National Natural Science Foundation of China (no. 81171811), the Special Scientific Research Projects of National Health and Family Planning Commission (no. 2015SQ00060), and the Natural Science Foundation of Shaanxi Province (no. 2014JM4180).

\section{References}

1. Singer AJ and Clark RA: Cutaneous wound healing. N Engl J Med 341: 738-746, 1999.

2. Santoro MM and Gaudino G: Cellular and molecular facets of keratinocyte reepithelization during wound healing. Exp Cell Res 304: 274-286, 2005.

3. Cary LA, Klinghoffer RA, Sachsenmaier C and Cooper JA: SRC catalytic but not scaffolding function is needed for integrin-regulated tyrosine phosphorylation, cell migration, and cell spreading. Mol Cell Biol 22: 2427-2440, 2002.

4. Avizienyte E, Fincham VJ, Brunton VG and Frame MC: Src $\mathrm{SH} 3 / 2$ domain-mediated peripheral accumulation of Src and phospho-myosin is linked to deregulation of E-cadherin and the epithelial-mesenchymal transition. Mol Biol Cell 15: 2794-2803, 2004.

5. González L, Agulló-Ortuño MT, García-Martínez JM, Calcabrini A, Gamallo C, Palacios J, Aranda A and Martín-Pérez J: Role of c-Src in human MCF7 breast cancer cell tumorigenesis. J Biol Chem 281: 20851-20864, 2006.

6. Ishizawar R and Parsons SJ: c-Src and cooperating partners in human cancer. Cancer Cell 6: 209-214, 2004.
7. Yoo SK, Freisinger CM, LeBert DC and Huttenlocher A: Early redox, Src family kinase, and calcium signaling integrate wound responses and tissue regeneration in zebrafish. J Cell Biol 199: 225-234, 2012.

8. Tsarouhas V, Yao L and Samakovlis C: Src kinases and ERK activate distinct responses to Stitcher receptor tyrosine kinase signaling during wound healing in Drosophila. J Cell Sci 127: 1829-1839, 2014.

9. Gao CY, Stepp MA, Fariss R and Zelenka P: Cdk5 regulates activation and localization of Src during corneal epithelial wound closure. J Cell Sci 117: 4089-4098, 2004.

10. Kähäri VM and Saarialho-Kere U: Matrix metalloproteinases in skin. Exp Dermatol 6: 199-213, 1997.

11. Madlener M, Parks WC and Werner S: Matrix metalloproteinases (MMPs) and their physiological inhibitors (TIMPs) are differentially expressed during excisional skin wound repair. Exp Cell Res 242: 201-210, 1998.

12. Ravanti L and Kähäri VM: Matrix metalloproteinases in wound repair (Review). Int J Mol Med 6: 391-407, 2000.

13. Coussens LM, Tinkle CL, Hanahan D and Werb Z: MMP-9 supplied by bone marrow-derived cells contributes to skin carcinogenesis. Cell 103: 481-490, 2000.

14. Lin CW, Chen PN, Chen MK, Yang WE, Tang CH, Yang SF and Hsieh YS: Kaempferol reduces matrix metalloproteinase-2 expression by down-regulating ERK $1 / 2$ and the activator protein-1 signaling pathways in oral cancer cells. PLoS One 8: e80883, 2013

15. Xue M, Le NT and Jackson CJ: Targeting matrix metalloproteases to improve cutaneous wound healing. Expert Opin Ther Targets 10: 143-155, 2006.

16. Asai J, Hirakawa S, Sakabe J, Kishida T, Wada M, Nakamura N, Takenaka H, Mazda O, Urano T, Suzuki-Inoue K, et al: Platelets regulate the migration of keratinocytes via podoplanin/CLEC-2 signaling during cutaneous wound healing in mice. Am J Pathol 186: 101-108, 2016.

17. Martin P: Wound healing - aiming for perfect skin regeneration. Science 276: 75-81, 1997.

18. Thomas SM and Brugge JS: Cellular functions regulated by Src family kinases. Annu Rev Cell Dev Biol 13: 513-609, 1997.

19. Raja, Sivamani K, Garcia MS and Isseroff RR: Wound re-epithelialization: modulating keratinocyte migration in wound healing. Front Biosci 12: 2849-2868, 2007.

20. Pilcher BK, Wang M, Qin XJ, Parks WC, Senior RM and Welgus HG: Role of matrix metalloproteinases and their inhibition in cutaneous wound healing and allergic contact hypersensitivity. Ann N Y Acad Sci 878: 12-24, 1999.

21. Pilcher BK, Dumin JA, Sudbeck BD, Krane SM, Welgus HG and Parks WC: The activity of collagenase-1 is required for keratinocyte migration on a type I collagen matrix. J Cell Biol 137: 1445-1457, 1997.

22. Sudbeck BD, Pilcher BK, Welgus HG and Parks WC: Induction and repression of collagenase-1 by keratinocytes is controlled by distinct components of different extracellular matrix compartments. J Biol Chem 272: 22103-22110, 1997.

23. Pilcher BK, Dumin J, Schwartz MJ, Mast BA, Schultz GS, Parks WC and Welgus HG: Keratinocyte collagenase-1 expression requires an epidermal growth factor receptor autocrine mechanism. J Biol Chem 274: 10372-10381, 1999.

24. Mirastschijski U, Haaksma CJ, Tomasek JJ and Agren MS: Matrix metalloproteinase inhibitor GM 6001 attenuates keratinocyte migration, contraction and myofibroblast formation in skin wounds. Exp Cell Res 299: 465-475, 2004.

25. Agren MS: Matrix metalloproteinases (MMPs) are required for re-epithelialization of cutaneous wounds. Arch Dermatol Res 291: 583-590, 1999.

26. Saarialho-Kere UK: Patterns of matrix metalloproteinase and TIMP expression in chronic ulcers. Arch Dermatol Res 290 (Suppl): S47-S54, 1998.

27. Fray MJ, Dickinson RP, Huggins JP and Occleston NL: A potent, selective inhibitor of matrix metalloproteinase-3 for the topical treatment of chronic dermal ulcers. J Med Chem 46: 3514-3525, 2003.

28. Yang Y, Bai ZG, Yin J, Wu GC and Zhang ZT: Role of c-Src activity in the regulation of gastric cancer cell migration. Oncol Rep 32: 45-49, 2014.

29. Perez-Moreno M, Jamora C and Fuchs E: Sticky business: orchestrating cellular signals at adherens junctions. Cell 112: $535-548,2003$.

30. Takeichi M: Cadherin cell adhesion receptors as a morphogenetic regulator. Science 251: 1451-1455, 1991. 
31. van Roy F and Berx G: The cell-cell adhesion molecule E-cadherin. Cell Mol Life Sci 65: 3756-3788, 2008.

32. Shindo M, Wada H, Kaido M, Tateno M, Aigaki T, Tsuda L and Hayashi S: Dual function of Src in the maintenance of adherens junctions during tracheal epithelial morphogenesis. Development 135: 1355-1364, 2008.

33. Dong S, Khoo A, Wei J, Bowser RK, Weathington NM, Xiao S, Zhang L, Ma H, Zhao Y and Zhao J: Serum starvation regulates E-cadherin upregulation via activation of c-Src in non-small-cell lung cancer A549 cells. Am J Physiol Cell Physiol 307: C893-C899, 2014.

34. Abreu-Blanco MT, Verboon JM, Liu R, Watts JJ and Parkhurst SM: Drosophila embryos close epithelial wounds using a combination of cellular protrusions and an actomyosin purse string. J Cell Sci 125: 5984-5997, 2012

35. Förster D and Luschnig S: Src42A-dependent polarized cell shape changes mediate epithelial tube elongation in Drosophila. Nat Cell Biol 14: 526-534, 2012.

36. Veracini L, Grall D, Schaub S, Beghelli-de la Forest Divonne S, Etienne-Grimaldi MC, Milano G, Bozec A, Babin E, Sudaka A, Thariat $\mathbf{J}$ and Van Obberghen-Schilling E: Elevated Src family kinase activity stabilizes E-cadherin-based junctions and collective movement of head and neck squamous cell carcinomas. Oncotarget 6: 7570-7583, 2014.

37. Deo DD, Axelrad TW, Robert EG, Marcheselli V, Bazan NG and Hunt JD: Phosphorylation of STAT-3 in response to basic fibroblast growth factor occurs through a mechanism involving platelet-activating factor, JAK-2, and Src in human umbilical vein endothelial cells. Evidence for a dual kinase mechanism. J Biol Chem 277: 21237-21245, 2002.

38. Goel R, Phillips-Mason PJ, Raben DM and Baldassare JJ: alpha-Thrombin induces rapid and sustained Akt phosphorylation by beta-arrestin1-dependent and -independent mechanisms, and only the sustained Akt phosphorylation is essential for G1 phase progression. J Biol Chem 277: 18640-18648, 2002.

39. Wu W, Graves LM, Gill GN, Parsons SJ and Samet JM: Src-dependent phosphorylation of the epidermal growth factor receptor on tyrosine 845 is required for zinc-induced Ras activation. J Biol Chem 277: 24252-24257, 2002.

40. Callera GE, Touyz RM, Tostes RC, Yogi A, He Y, Malkinson S and Schiffrin EL: Aldosterone activates vascular p38MAP kinase and NADPH oxidase via c-Src. Hypertension 45: 773-779, 2005.

41. Khadaroo RG, He R, Parodo J, Powers KA, Marshall JC, Kapus A and Rotstein OD: The role of the Src family of tyrosine kinases after oxidant-induced lung injury in vivo. Surgery 136 483-488, 2004.
42. Khadaroo RG, Parodo J, Powers KA, Papia G, Marshall JC, Kapus A and Rotstein OD: Oxidant-induced priming of the macrophage involves activation of $\mathrm{p} 38$ mitogen-activated protein kinase through an Src-dependent pathway. Surgery 134: 242-246, 2003.

43. Chen Y, Ramakrishnan DP and Ren B: Regulation of angiogenesis by phospholipid lysophosphatidic acid. Front Biosci (Landmark Ed) 18: 852-861, 2013

44. He M, Xue ZM, Li J and Zhou BQ: Breviscapine inhibits high glucose-induced proliferation and migration of cultured vascular smooth muscle cells of rats via suppressing the ERK1/2 MAPK signaling pathway. Acta Pharmacol Sin 33: 606-614, 2012.

45. Makino T, Jinnin M, Muchemwa FC, Fukushima S, KogushiNishi H, Moriya C, Igata T, Fujisawa A, Johno T and Ihn H: Basic fibroblast growth factor stimulates the proliferation of human dermal fibroblasts via the ERK1/2 and JNK pathways. Br J Dermatol 162: 717-723, 2010.

46. Yue GG, Fan JT, Lee JK, Zeng GZ, Ho TW, Fung KP, Leung PC, Tan NH and Lau CB: Cyclopeptide RA-V inhibits angiogenesis by down-regulating ERK1/2 phosphorylation in HUVEC and HMEC-1 endothelial cells. Br J Pharmacol 164: 1883-1898, 2011.

47. Gazel A, Nijhawan RI, Walsh R and Blumenberg M: Transeriptional profiling defines the roles of ERK and p38 kinases in epidermal keratinocytes. J Cell Physiol 215: 292-308, 2008.

48. Boulton TG, Nye SH, Robbins DJ, Ip NY, Radziejewska E, Morgenbesser SD, DePinho RA, Panayotatos N, Cobb MH and Yancopoulos GD: ERKs: a family of protein-serine/threonine kinases that are activated and tyrosine phosphorylated in response to insulin and NGF. Cell 65: 663-675, 1991.

49. Kim MS, Kim YK, Eun HC, Cho KH and Chung JH: All-trans retinoic acid antagonizes UV-induced VEGF production and angiogenesis via the inhibition of ERK activation in human skin keratinocytes. J Invest Dermatol 126: 2697-2706, 2006.

50. Matsubayashi Y, Ebisuya M, Honjoh S and Nishida E: ERK activation propagates in epithelial cell sheets and regulates their migration during wound healing. Curr Biol 14: 731-735, 2004.

51. Ennis BW, Fultz KE, Smith KA, Westwick JK, Zhu D, Boluro-Ajayi M, Bilter GK and Stein B: Inhibition of tumor growth, angiogenesis, and tumor cell proliferation by a small molecule inhibitor of c-Jun N-terminal kinase. J Pharmacol Exp Ther 313: 325-332, 2005.

52. Harper EG, Alvares SM and Carter WG: Wounding activates p38 map kinase and activation transcription factor 3 in leading keratinocytes. J Cell Sci 118: 3471-3485, 2005. 